\title{
Protein binder woes
}

\author{
The biology research landscape is littered with antibodies that perform poorly for \\ many applications. Two publicly funded academic efforts to explore high-quality binder \\ generation are coming to an end. What have we learned-and what happens next?
}

Protein binders-reagents such as antibodies that bind a specific target protein-have a reach both deep and wide in biological research. They may be used to label and image a protein within tissue, to isolate cells on the basis of marker expression, or to physically capture a protein from a complex biological mixture, to name just a few applications. Binders are essential tools for virtually every biological research discipline, and in every biological system.

For such important tools, one would expect to have a system in place that consistently delivers high-quality reagents. Lamentably, this is not the case. Systematic studies of antibody performance, specific cases of performance problems (such as the recent debate over the protein irisin), and the anecdotal experiences of researchers in many disciplines lead to the conclusion that many binders perform suboptimally and may often not be used with enough care. Poor antibodies may well contribute to the now much-discussed irreproducibility of some published results.

The problem of antibody performance is complex, for many reasons. The definition of a high-quality binder is very context and application dependent. Strong and specific binding in vitro does not necessarily predict performance in blood, tissue or electrophoretically-separated lysate, for example, where epitope presence or accessibility as well as relative target concentration may vary. It is not trivial to validate a binder for all possible applications, and even a wellcharacterized tool may perform suboptimally in a new context. This does not mean that validation should not be done, just that it cannot be perfect.

Furthermore, as recently pointed out in Nature, the identity of commercially available research antibodies is often distressingly murky. The same reagents may be sold under different names, or, conversely, a particular identifier may not always deliver the same binder. On top of this, a culture persists in which too many commercial binders simply do not work well, and researchers continue to purchase suboptimal reagents.

Two publicly funded consortia have explored the feasibility of generating high-quality renewable binder resources in a not-for-profit setting. In the United States, the National Institutes of Health (NIH) Common Fund's Protein Capture Reagents program, which began in 2010, focused on binders to transcription factors and chromatin-associated proteins. The European Union-funded Affinomics consortium, which began as ProteomeBinders and set out its initial manifesto in our pages in 2007, focused on generating binders to various signaling proteins and to some cancer markers. Both consortia are composed of research groups involved in antigen production, binder selection or binder validation. Their remit has also included technology development for generating recombinant binders.

Affinomics and the NIH program have both generated accessible collections of validated binders. The NIH Protein Capture site reports 854 binders against 162 proteins, most of them either mouse monoclonal antibodies or recombinant antibodies selected by phage display. The Affinomics Protein Binders site reports 5,408 binders against 2,900 antigens; again the binders may be conventional antibodies or recombinant tools, including nanobodies, single chain antibody fragments, and alternative scaffolds based on designed ankyrin repeats.

Both consortia emphasize tool validation, but as a perfect validation pipeline is unrealistic, it will remain important to assess how these tools perform in the wider community. Also, although recombinant binders can help solve the problems of renewability and identity, the need for performance validation remains.

Perhaps most importantly, the consortia have shown that it is feasible for collaborative academic groups to generate and validate binders, in high throughput, against a set of target proteins, and to do so using traditional and recombinant technologies in parallel. This said, there almost certainly remains room for methodological improvement, including reducing costs for recombinant pipelines.

Both pilot projects are now winding down, and it is disheartening that there are no immediate consortiumlevel plans, or funds, for harnessing their experience and pipelines (whether in present or improved form) to continue to generate binders for the community. Especially given the many inadequacies of the current market-driven system, developments in these directions and exploration of sustainable routes for binder generation and production will be critical. Binders are just too important, for too much of biology, to let these efforts fade away. 